\title{
Effect of Heel Height on the Foot in Unilateral Standing
}

\author{
Mika Shimizu1), Paul D. Andrew2) \\ 1) Department of Rehabilitation, Shimonoseki Daiichi Hospital, 9-6 Imaura-cho, \\ Shimonoseki 750-0064, Japan. TEL +81 832-32-5811 \\ ${ }^{2)}$ Division of Physical Therapy, Institute of Health Sciences, Faculty of Medicine, \\ Hiroshima University
}

\begin{abstract}
The purpose of this investigation was to study the effect of elevating the heel during unilateral weight-bearing on structure of the foot, especially a "flat" foot, and on function of the foot in maintaining balance. We examined 16 healthy women, 21 to 23 years of age, who stood unilaterally on the right foot with the heel elevated $0,5,10,15,20,25,30$, and $40 \mathrm{~mm}$. We measured height of the medial longitudinal arch, rearfoot pronation (calf-to-calcaneus angle), displacement of center of pressure under the foot during ten seconds, and mean position of the center of pressure during those ten seconds. Elevating the heel raised the medial longitudinal arch, reduced rearfoot pronation, increased length of displacement of the center of pressure, and brought the mean center of pressure forward and medially. In feet that initially had strong rearfoot pronation, presence of an initially high medial longitudinal arch was associated with a greater curtailment of rearfoot pronation when the heel was elevated than if the arch was not initially high. These results suggest that elevating the heel of a shoe can help reduce the signs of a flat foot, but the accompanying decrease in balance stability and shift of pressure toward the first metatarsal head makes the use of elevated heels questionable as a therapeutic measure.
\end{abstract}

Key words: Heel height, Rearfoot pronation, Unilateral standing balance.

(This article was submitted May 6, 1999, and accepted Sep. 20, 1999)

\section{INTRODUCTION}

Although shoes play a very important role in the practical aspects of daily life, fashion has influenced their design to the point that, in some instances, the practical function of shoes becomes compromised. For example, many women wear shoes with high heels, presumably to increase their stature in the eyes of men. As they walk with high heels, however, the weight usually borne with each step by the forefoot becomes excessively concentrated in the region of the metatarsal heads ${ }^{1,2)}$, particularly the first metatarsal head ${ }^{1)}$. Habitual loading of this nature can lead to such disorders as hallux valgus ${ }^{3}$.

We were interested in what effect high heels might have on flat feet, given that such shoes would induce hyperextension of the toes, thereby activating the "windlass mechanism"4) in the foot, which in turn would increase the height of the medial longitudinal arch. This question, however, must be preceded by a discussion about what constitutes a "flat" foot and other related issues.

First, the calf-to-calcaneus angle is the angle by which the vertical orientation of the calcaneus deviates into eversion from the long axis of the calf. A large calf-to-calcaneus angle, say eight degrees or so, is typically associated with a pronated foot. For purposes of this paper, eversion means movement in the frontal plane of the foot in which the plantar surface is tilted away from the midline of the body, whereas pronation is a natural combination of dorsiflexion, eversion, and abduction of the 
foot. Because of the complexity of pronation, one can refer to forefoot pronation or rearfoot pronation as separate movements. Rearfoot pronation takes place primarily in the subtalar joint and is generally assessed by measuring the calf-to-calcaneus angle. In this paper, rearfoot pronation should be considered in this specific sense of a positive calf-to-calcaneus angle.

Rearfoot pronation, in turn, is typically associated with flatfoot deformity or, more specifically, forefoot varus ${ }^{5)}$. The link between calcaneal eversion and flat foot can be found in the oblique nature of the axis of the subtalar joint which, as one goes from posterior to anterior, runs from lateral to medial and from superior to inferior. Hicks ${ }^{6}$ ) demonstrated that by virtue of this axis lateral rotation of the tibia would move the calcaneus in the direction of inversion and increase the height of the medial longitudinal arch of the foot. Hicks ${ }^{4)}$ further showed that hyperextension of the toes, especially the great toe, pulls the plantar connective tissue about the metatarsal heads like a windlass and thereby increases the height of the medial longitudinal arch and thus slightly inverts the position of the calcaneus.

Because the toes are forced into hyperextension when high heels are worn, the medial longitudinal arch of a foot in a high-heeled shoe can be expected to become more pronounced. What kind of effect might this have on balance function? High heels have been shown to alter posture slightly. The lumbar lordosis is decreased ${ }^{7-9)}$ and the ankle and knee joints are brought closer to the vertical line that passes through the body's center of gravity ${ }^{8,9)}$. Constraining the calcaneus into slight inversion or eversion, without elevating the heel, has been shown to reduce mediolateral sway when the subject stands on one foot, and to shift weight toward the great toe if the constraint is in inversion ${ }^{10)}$.

In the present study we examined the effect of elevating the heel of the right foot on structure (changes in calf-to-calcaneus angle and in height of the medial longitudinal arch during right singleleg stance) and on balance function (change in length of displacement of center of pressure over ten seconds of standing on the right leg).

\section{METHODS}

Sixteen healthy women 21 to 23 years of age participated in this study. The right heel was placed on $60 \times 70 \mathrm{~mm}$ cork plates of various heights $(5,10,15,20,25,30$, and $40 \mathrm{~mm})$ as well as simply on the floor (height of $0 \mathrm{~mm}$ ). Calf-tocalcaneus angle and height of the medial longitudinal arch were measured while the subject was standing only on the right foot at the different heel heights.

Height of the medial longitudinal arch was determined as the height of the navicular tuberosity from a line joining the lowest part of the calcaneal tuberosity and the medial sesamoid bone at the distal part of the first metatarsal. At each height of the heel, this measurement was taken three times. For purposes of classifying the subjects' feet, we also calculated a normalized initial arch height in which the height of the medial longitudinal arch at no elevation was divided by the length of the foot $^{11)}$.

The calf-to-calcaneus angle of the right foot was measured three times at each of the eight heel heights with the subject standing on only the right foot. One straight line was drawn down the middle of the right calf and another along the posteriormost surface of the right calcaneus to indicate the orientations of these two segments. We used a goniometer to measure the angle between these two lines. This procedure is derived from a description by Elveru et al ${ }^{12}$.

Length of displacement of center of pressure over ten seconds was measured by a device especially designed for this purpose (Gravicorder GS-10, type C IV, Anima Ltd., Naruko Bldg., Nishi-shinjuku 8-4-1, Shinjuku-ku, Tokyo 1600023). The device was a triangular platform on which footprints were drawn to indicate proper position and orientation of the feet for bilateral standing. Output from a wire strain gauge at each corner of the platform was processed to calculate the center of pressure applied to the platform. Within the device itself the displacement data were sampled at 20 samples per second. The length of displacement of the center of pressure over ten seconds was calculated as

$$
\sum_{i=2}^{200} \sqrt{\left(x_{i}-x_{i-1}\right)^{2}+\left(y_{i}-y_{i-1}\right)^{2}}
$$

where $x_{i}$ is the mediolateral location of the ith sample of center of pressure, $y_{i}$ is the corresponding anteroposterior location, and 200 is the number of samples collected over ten seconds. The device also calculated the mean mediolateral and anteroposterior locations of the center of pressure over 
the sampling period of ten seconds.

For each measurement the subject had to have been standing on the right foot for ten seconds before the sampling of an additional ten seconds began. While the subject stood on the right foot, she looked at a circle $50 \mathrm{~mm}$ in diameter placed three meters in front of her at eye level. Two successive measurements were taken at each heel height. The device was calibrated before each heel height was measured to account for the pressure exerted by the cork plate for that heel height.

We used analyses of variance with repeated measures and product moment correlations to examine the effect of heel height on arch height, calf-tocalcaneus angle, length of displacement of center of pressure over ten seconds, and mean mediolateral and anteroposterior locations of the center of pressure of the foot over the same period of ten seconds. To assess reliability of the measurements used in this study, we randomly chose four subjects to go through the identical procedures on a different day. Intrasession reliability was based on three repetitions of each measurement of arch height and of calf-to-calcaneus angle, and on two repetitions of the measurements for center of pressure. Intersession reliability was based on all of these measurements being assessed again on a second day in the four subjects. We calculated intraclass correlation coefficients $(\operatorname{ICC}[3,1])^{13)}$ and standard errors of measurement.

\section{RESULTS}

Analysis of variance indicated that heel height had a highly significant $(p<0.00001)$ effect on each variable measured. Considerable variations among the subjects, however, reduced the sharpness of the product moment correlations. Because of the variety of feet seen in the sample, we categorized them into four types on the basis of normalized initial arch height and calf-to-calcaneus angle with no heel elevation. Normalized initial arch height, determined by dividing the height of the navicular tuberosity by the length of the foot during standing on a flat surface ${ }^{11}$, was $17.98 \% \pm 2.17 \%$ (mean \pm standard deviation) in this sample. The calf-tocalcaneus angle with no heel elevation was $8.36^{\circ} \pm$ $5.47^{\circ}$. Using the sample means of these two measurements as criteria for classification, the subjects' feet were categorized into four types as shown in Table 1.
Table 1. Categorization of subjects by foot structure

\begin{tabular}{ccc}
\hline & \multicolumn{2}{c}{ Normalized Arch Height } \\
Rearfoot Pronation & Low $(<18 \%)$ & High $(>18 \%)$ \\
\hline Weak $\left(<8^{\circ}\right)$ & 4 subjects & 4 subjects \\
Strong $\left(>8^{\circ}\right)$ & 5 subjects & 3 subjects \\
\hline
\end{tabular}

As height of the heel increased, height of the medial longitudinal arch increased (Fig. 1, top) and the calf-to-calcaneus angle decreased (Fig. 1, middle). The middle panel of Figure 1 reveals that normalized initial arch height had little influence on calf-to-calcaneus angles that initially had relatively little eversion (triangles), but high normalized initial arch height appeared to be associated with curtailed eversion in feet that initially had relatively large calf-to-calcaneus angles (empty circles). Unfortunately, the small sizes of the subgroups made post hoc use of statistical inference infeasible.

Length of displacement of center of pressure over ten seconds increased with heel height (Fig. 1, bottom). Although type of foot did not strongly influence the nature of the results, the feet with strong rearfoot pronation (circles) tended to have greater displacements than did the other feet, regardless of normalized arch height. As height of the heel increased, location of the center of pressure migrated medially (Fig. 2, top) and, after having overcome a threshold at $15 \mathrm{~mm}$, it progressed forward (Fig. 2, bottom).

Reliabilities of the measures analyzed in this study are summarized in Table 2 . Intersession reliability was essentially the same as intrasession reliability for all phenomena measured, suggesting that these variables would be stable from one session to another. Calf-to-calcaneus angle and arch height were both reproducible and precise, but the measures related to center of pressure at the foot were quite variable in nature.

\section{DISCUSSION}

That the medial longitudinal arch increased and the calf-to-calcaneus angle decreased as heel height was raised agrees with the idea of the windlass mechanism in the foot ${ }^{4}$. As the great toe extends, the plantar fascia is stretched, consequently increasing height of the arch. This function of the plantar fascia in the windlass mechanism was re- 

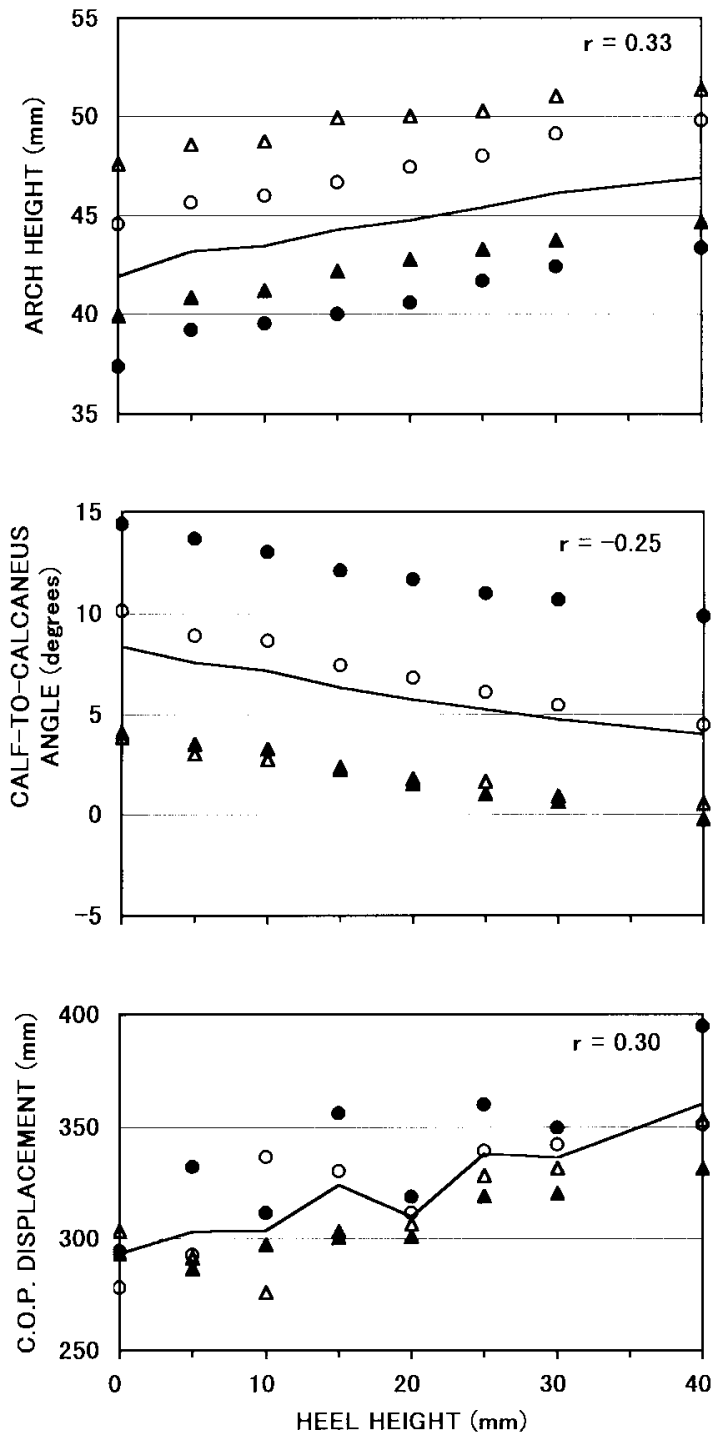

Fig. 1. Changes in height of arch (top), calf-tocalcaneus angle (middle), and length of displacement of center of pressure during ten seconds of unilateral weight-bearing (bottom) in response to elevation of the heel. Empty symbols represent averages of subjects with high arches, filled symbols averages of subjects with low arches. Triangles represent averages of subjects with weak rearfoot pronation, circles averages of subjects with strong rearfoot pronation. The continuous line shows the overall mean values. The correlation coefficient $(r)$ indicates overall correlation between height of the heel and the average measurement per subject of the variable on the ordinate.
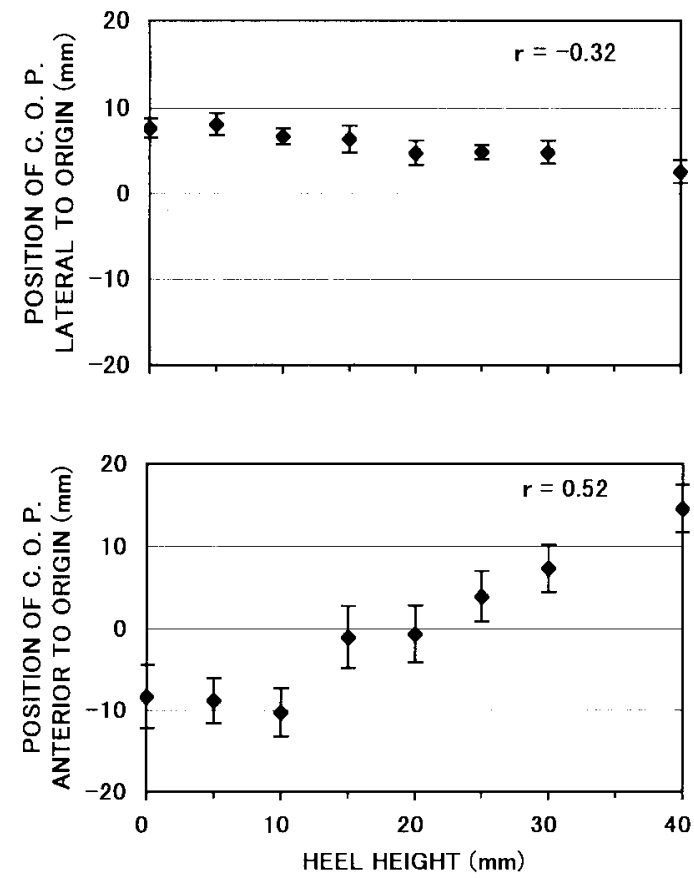

Fig. 2. Effect of heel height on mean position of center of pressure (and standard error) in mediolateral (top) and anteroposterior (bottom) directions. Values of zero simply indicate origin of the coordinate system on the measurement platform, so the important information in these graphs is change in position as height of the heel varies. The correlation coefficient $(r)$ indicates overall correlation between height of the heel and the average measurement per subject of the variable on the ordinate.

cently verified in a study on cadaver specimens in which the plantar fascia was sectioned to varying extents ${ }^{14)}$. In each cycle of gait, the height of the medial longitudinal arch increases as the toes hyperextend ${ }^{15)}$. High heels curtail the extent to which the arches flatten during the gait cycle ${ }^{16}$.

In the present study, elevating the heel more powerfully constrained rearfoot pronation in subjects with strongly pronated feet if the subject also had a relatively high arch to begin with. This finding appears to reflect the function of a tightly biased windlass mechanism in the foot with an initially high arch.

Increase in height of the medial longitudinal arch was accompanied by decrease in rearfoot pronation. Both of these changes are associated with decrease of flat foot and are interrelated ${ }^{4,6)}$ as ex- 
Table 2. Reliability of measurements performed in this study

\begin{tabular}{lccccc}
\hline & \multicolumn{2}{c}{ Intraclass Correlation Coefficient } & & \multicolumn{2}{c}{ Standard Error of Measurement } \\
\cline { 2 - 3 } & Intrasession & Intersession & & Intrasession & Intersession \\
\hline Calf-to-Calcaneus Angle & 0.95 & 0.94 & & $1.09^{\circ}$ & $1.10^{\circ}$ \\
Arch Height & 0.81 & 0.80 & & $1.99 \mathrm{~mm}$ & $2.03 \mathrm{~mm}$ \\
COP Displacement & 0.46 & 0.33 & & $60.8 \mathrm{~mm}$ & $68.1 \mathrm{~mm}$ \\
Mean COP Mediolateral Position & 0.35 & 0.35 & & $5.9 \mathrm{~mm}$ & $5.9 \mathrm{~mm}$ \\
Mean COP Anteroposterior Position & 0.40 & 0.37 & & $11.6 \mathrm{~mm}$ & $11.9 \mathrm{~mm}$ \\
\hline
\end{tabular}

COP: center of pressure

plained in the introduction. Shift of the center of pressure forward and medially as the heel was elevated is consistent with findings by Corrigan et $\mathrm{al}^{1)}$. Use of heels thus appears to reduce signs of a flat foot, but also shifts pressure toward the first metatarsal head and tends to make balance more difficult during unilateral weight-bearing.

When high heels are not worn, muscles do not appear to play an important role in holding the arches. Basmajian and Stecko ${ }^{17)}$ established that the arch of the foot can be maintained without muscular activity even when the arch is fairly heavily loaded. Extrinsic muscles that cross under the plantar surface, notably the peroneus longus and tibialis posterior, are capable of enhancing the height of the medial longitudinal arch, but they are not ordinarily necessary to maintain the arch.

Elevating the heel complicates the situation. Lee et al found that elevating the heels decreases activity in the gastrocnemius but increases activity in the tibialis anterior during walking in men ${ }^{18)}$, whereas activity in both muscles decreases under similar circumstances in women ${ }^{19)}$. Joseph and Nightingale ${ }^{20}$ ) found that raising the heels in women increases activity in the soleus, but not in other muscles. Basmajian and Bentzon ${ }^{21)}$ noted more activity in the gastrocnemius and peroneus longus in response to elevating the heels $63 \mathrm{~mm}$, but not in other muscles. Ono ${ }^{22)}$ obtained results similar to those of Basmajian and Bentzon ${ }^{21)}$, but not if the height of the heels was less than $50 \mathrm{~mm}$.

In flat feet, muscles may play a greater role in trying to support a medial longitudinal arch. Suzuki ${ }^{23)}$ noted increased activity in the peroneus longus in flat feet, while Gray and Basmajian ${ }^{24)}$ saw what they interpreted to be reflex activity during gait in flat feet from the tibialis anterior, tibialis posterior, and peroneus longus.

The role of muscular activity is thus questionable as it relates to our results concerning height of the arch or calf-to-calcaneus angle, but the greater displacement of center of pressure in the flatter feet (those with relatively large calf-to-calcaneus angles), whether or not the heels were elevated, can best be explained by enhanced muscular activity.

As far as we have been able to determine, relations among arch height, rearfoot pronation, balance, and imposed heel height remain inadequately investigated. Because the length of displacement of center of pressure did not yield very precise results in this study, we believe that other ways to assess unilateral standing balance should be considered. We recommend further investigation into the extent to which the windlass mechanism alters configuration of the medial longitudinal arch in flat feet versus normal feet, as well as studies on electromyographic activities of muscles about the ankle and foot joints during unilateral balance with the heel at various heights.

\section{CONCLUSION}

Our results suggest that elevating the heel of a foot tends to increase height of the medial longitudinal arch, reduce rearfoot pronation, lengthen the displacement of center of foot pressure during unilateral weight-bearing, and bring that center of foot pressure toward the first metatarsal head. We thus see that whereas elevating the heel tends to attenuate the structural signs of a flat foot, the accompanying changes in balance function make one hesitate to recommend heel elevation as an antidote for flat feet.

\section{REFERENCES}

1) Corrigan JP, Moore DP, Stephens MM: Effect of heel height on forefoot loading. Foot Ankle, 1993, 14: 
$148-152$.

2) Snow RE, Williams KR, Holmes GB: The effects of wearing high heeled shoes on pedal pressure in women. Foot Ankle, 1992, 13: 85-92.

3) Nyska M, McCabe C, Linge K, et al.: Plantar foot pressures during treadmill walking with high-heel and low-heel shoes. Foot Ankle Int, 1996, 17: 662-666.

4) Hicks JH: The mechanics of the foot: II. The plantar aponeurosis and the arch. J Anat, 1954, 88: 25-30.

5) Hlavac HF: Compensated forefoot varus. J Am Podiatr Assoc, 1970, 60: 229-233.

6) Hicks JH: The mechanics of the foot: I. The joints. J Anat, 1953, 87: 345-357.

7) de Lateur BJ, Giaconi RM, Questad K, et al.: Footwear and posture: Compensatory strategies for heel height. Am J Phys Med Rehabil, 1991, 70: 246-254.

8) Opila KA, Wagner SS, Schiowitz S, et al.: Postural alignment in barefoot and high-heeled stance. Spine, 1988, 13: 542-547.

9) Bendix T, Sorensen SS, Klausen K: Lumbar curve, trunk muscles, and line of gravity with different heel heights. Spine, 1984, 9: 223-227.

10) Suzuki S, Iritani M, Ohno N, et al.: Effect of change in heel position of subtalar joint on standing balance: analysis of body sway directions. J Phys Ther Sci, 1992, 4: 109-113.

11) Okubo M, Shimazu A, Ueno K, et al.: Investigation of method to measure foot arch height in medical check. Rinsho Supotsu Igaku, 1989, 6 (Bessatsu): 336-339 (in Japanese).

12) Elveru RA, Rothstein JM, Lamb RL, et al.: Methods for taking subtalar joint measurements: a clinical report. Phys Ther, 1988, 68: 678-682.

13) Shrout PE, Fleiss JL: Intraclass correlations: uses in assessing rater reliability. Psychol Bull, 1979, 86: 420-428.

14) Thordarson DB, Kumar PJ, Hedman TP, et al.: Effect of partial versus complete plantar fasciotomy on the windlass mechanism. Foot Ankle Int, 1997, 18: 1620.

15) Kayano J: Dynamic function of medial foot arch. Nippon Seikeigeka Gakkai Zasshi, 1986, 60: 11471156.

16) Teramoto T: On heel height of shoe and foot medial arch. Nagasaki Igakkai Zasshi, 1987, 62: 435-447 (in Japanese).

17) Basmajian JV, Stecko G: The role of muscles in arch support of the foot. J Bone Joint Surg Am, 1963, 45: 1184-1190.

18) Lee KH, Matteliano A, Medige J, et al.: Electromyographic changes of leg muscles with heel lift: therapeutic implications. Arch Phys Med Rehabil, 1987, 68: 298-301.

19) Lee KH, Shieh JC, Matteliano A, et al.: Electromyographic changes of leg muscles with heel lifts in women: therapeutic implications. Arch Phys Med Rehabil, 1990, 71: 31-33.

20) Joseph J, Nightingale A: Electromyography of muscles of posture: leg and thigh muscles in women, including the effects of high heels. J Physiol, 1956, 132: 465-468.

21) Basmajian JV, Bentzon JW: An electromyographic study of certain muscles of the leg and foot in the standing position. Surg Gynec Obstet, 1954, 98: 662666.

22) Ono H: Heel height and muscle activity. Nippon Seikeigeka Gakkai Zasshi, 1969, 43: 527-541 (in Japanese).

23) Suzuki N: An electromyographic study of the role of muscles in arch support of the normal and flat foot. Nagoya Med J, 1972, 17: 57-79.

24) Gray ER, Basmajian JV: Electromyography and cinematography of leg and foot ("normal" and flat) during walking. Anat Rec, 1968, 161: 1-15. 